\title{
Predicting Adherence to Chronic Disease Medications in Patients with Long-term Initial Medication Fills Using Indicators of Clinical Events and Health Behaviors
}

\author{
Julie C. Lauffenburger, PharmD, PhD; Jessica M. Franklin, PhD; Alexis A. Krumme, ScD; \\ William H. Shrank, MD, MSHS; Olga S. Matlin, PhD; Claire M. Spettell, PhD; Gregory Brill, MS; \\ and Niteesh K. Choudhry, MD, PhD
}

\begin{abstract}
BACKGROUND: Efforts at predicting long-term adherence to medications have been focused on patients filling typical month-Iong supplies of medication. However, prediction remains difficult for patients filling longer initial supplies, a practice that is becoming increasingly common as a method to enhance medication adherence.
\end{abstract}

OBJECTIVES: To (a) extend methods involving short-term filling behaviors and (b) develop novel variables to predict adherence in a cohort of patients receiving longer initial prescriptions.

METHODS: In this retrospective cohort study, we used claims from a large national insurer to identify patients initiating a 90-day supply of oral medications for diabetes, hypertension, and hyperlipidemia (i.e., statins). Patients were included in the cohort if they had continuous database enrollment in the 180 days before and 365 days after medication initiation. Adherence was measured in the subsequent 12 months using the proportion of days covered metric. In total, 125 demographic, clinical, and medication characteristics at baseline and in the first 30-120 days after initiation were used to predict adherence using logistic regression models. We used 10 -fold cross-validation to assess predictive accuracy by discrimination (c-statistic) measures.

RESULTS: In total, 32,249 patients met the inclusion criteria, including 14,930 patients initiating statins, 12,887 patients initiating antihypertensives, and 4,432 patients initiating oral hypoglycemics. Prediction using only baseline variables was relatively poor (cross-validated c-statistic = 0.644). Including indicators of acute clinical conditions, health resource utilization, and short-term medication filling in the first 120 days greatly improved predictive ability $(0.823)$. A model that incorporated all baseline characteristics and predictors within the first 120 days after medication initiation more accurately predicted future adherence (0.832). The best performing model that included all 125 baseline and postbaseline characteristics had strong predictive ability $(0.837)$, suggesting the utility of measuring these novel postbaseline variables in this population.

CONCLUSIONS: We demonstrate that long-term, 12-month adherence in patients filling longer supplies of medication can be strongly predicted using a combination of clinical, health resource utilization, and medication filling characteristics before and after treatment initiation.

J Manag Care Spec Pharm. 2018;24(5):469-77

Copyright $\odot 2018$, Academy of Managed Care Pharmacy. All rights reserved.

\section{What is already known about this subject}

An increasingly large number of patients at retail and mail order pharmacies are being provided with more medication per fill, generally 90 days, so that fewer refills are necessary, with the aim of increasing medication adherence.

While rates of adherence are generally slightly higher among patients with 90-day medication supplies than with 30-day supplies, adherence is still low and the ability of payer organizations to predict adherence among patients who are initiating these long initial medication fills has been very limited.

\section{What this study adds}

Models predicting medication adherence using variables measured in the first 120 days after medication initiation, such as indicators of adherence, refilling shortly after initiation, rates of new clinical events, and health resource utilization were much more accurate compared with models that used patient baseline information alone.

Long-term adherence among the growing subgroup of patients filling longer supplies of medication can be strongly predicted using a combination of characteristics before and shortly after treatment initiation as measured in this study.

Efforts to predict adherence among patients initiating medications with long-term ( $\geq 90$ days) initial supplies for potential intervention may benefit from including these predictors.

L ong-term adherence to evidence-based medications for chronic diseases remains poor. ${ }^{1}$ Only 1 year after beginning therapy, less than $50 \%$ of patients are still taking their medications as prescribed. ${ }^{2}$ Poor adherence leads to substantial increases in preventable and costly clinical outcomes. ${ }^{3,4}$ Because adherence is a key quality measure for health plans and providers and affects quality ratings and performance incentives, additional tools are needed to predict and ultimately to provide interventions for individuals at risk of nonadherence.

Advances have been made at predicting long-term adherence to medications but have been mainly concentrated on 


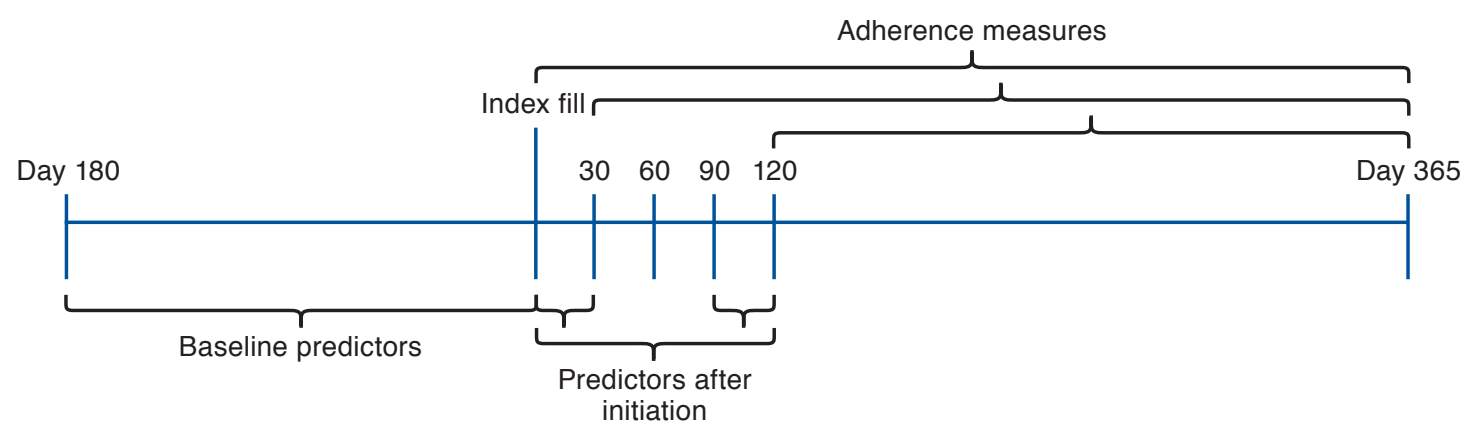

patients who fill typical month-long supplies of medication.5-9 At the same time, patients are increasingly being provided with more medication per fill, generally 90 days, so that fewer refills are necessary; these longer-term fills are available through both mail order and retail pharmacies. ${ }^{10-12}$ For these individuals, adherence prediction remains problematic. ${ }^{5,6}$ Initial patterns of refill behavior have been shown to substantially improve prediction accuracy among patients with month-long dispensations, indicating that short-term adherence behaviors could be used to predict future adherence. ${ }^{6}$ However, extending the time window over which initial filling patterns are observed could improve predictive accuracy for 90-day fillers but comes at the expense of waiting longer to initiate interventions designed to help individuals who are highly likely to be nonadherent in the future.

Accordingly, we sought to identify which, if any, of the existing methods of adherence prediction among month-long fillers could accurately and practically be applied in the 90-day filling context. We aimed to supplement these techniques with novel approaches for predicting adherence using other health behaviors measured shortly after initiation among this increasingly important group of individuals.

\section{Methods}

\section{Data Source and Patient Population}

We identified patients initiating an oral medication for diabetes or hypertension or a statin for hyperlipidemia between January 1 , 2012, and December 31, 2013, from an administrative dataset of medical and prescription claims data for commercial, fully insured members of Aetna, a large national health insurer. This Health Insurance Portability and Accountability Act limited dataset includes complete patient-level claims for all procedures, physician encounters, hospitalizations, and prescriptions and was also linked to enrollment data including patient age, gender, and ZIP code of residence. Aggregate data on socioeconomic status, race/ethnicity, and educational attainment for each ZIP code were obtained by linking the patient's residing ZIP code with data from the 2010 U.S. census. The insti- tutional review board of the Brigham and Women's Hospital approved this study.

The study medication list was drawn from the Health Effectiveness Data and Information tables of oral medications for that disease state. ${ }^{13}$ Each patient's first fill date for any study medication was considered to be the "index date." New initiation was defined as not filling a medication for that therapeutic class in the previous 180 days; patients using insulin who met these criteria were not excluded. For patients who filled $>1$ eligible medication, we considered only the medication with the earliest index date.

We included patients who were $\geq 18$ years of age, had nonmissing age, gender, and ZIP code information $(<1 \%$ missing for all fields), and had $\geq 1$ drug claim and $\geq 1$ medical claim and continuous enrollment in medical and pharmacy benefits in the 180-day period before the index date to ensure active plan benefit use. We also required patients to have continuous database enrollment in the 365-day period after the index date. By definition, the patients included in the final cohort had no other missing data as they had complete pharmacy and medical claims for the baseline and follow-up measurement periods. To focus on individuals filling medications with longer days supplied, patients were excluded if the days supply of their index fill was <90 days. The overall study design is shown in Figure 1 .

\section{Adherence Measures}

The outcome of interest was adherence to medications for 1 of 3 therapeutic conditions over the 12 months after medication initiation. For each medication, we created a drug supply diary linking the days of medication supplied for all observed fills from the index date through the next 365 days based on dispensing date and days supplied. Different medications in the same chemically related therapeutic class (e.g., beta-blockers) were considered to be interchangeable. We chose to evaluate adherence at the therapeutic class level, as was done frequently in prior research and quality improvement efforts. This method 


\section{Predicting Adherence to Chronic Disease Medications in Patients with Long-term Initial Medication Fills Using Indicators of Clinical Events and Health Behaviors}

\section{TABLE 1 Baseline Characteristics of the Study Cohort}

\begin{tabular}{|c|c|c|c|c|c|}
\hline Characteristic, \% & $\begin{array}{l}\text { Fully Adherent }^{\mathrm{a}} \\
\mathrm{n}=12,068\end{array}$ & $\begin{array}{l}\text { Poorly Adherent }{ }^{\mathrm{a}} \\
\mathrm{n}=20,181\end{array}$ & Characteristic, \% & $\begin{array}{l}\text { Fully Adherent }{ }^{\mathrm{a}} \\
\mathrm{n}=12,068\end{array}$ & $\begin{array}{c}\text { Poorly Adherent }{ }^{\mathrm{a}} \\
\mathrm{n}=20,181\end{array}$ \\
\hline Hyperlipidemia cohort & 37.6 & 62.4 & \multicolumn{3}{|l|}{ Clinical } \\
\hline Hypertension cohort & 42.8 & 57.2 & Peripheral vascular disease & 0.2 & 0.2 \\
\hline Diabetes cohort & 35.6 & 64.4 & Stroke/TIA & 0.2 & 0.2 \\
\hline \multicolumn{3}{|l|}{ Demographic } & Allergic reaction & 0.9 & 0.9 \\
\hline Female & 41.0 & 44.6 & \multirow{2}{*}{$\begin{array}{l}\text { Coronary artery bypass graft } \\
\text { surgery }\end{array}$} & \multirow[t]{2}{*}{0.1} & \multirow[t]{2}{*}{0.1} \\
\hline Age, years, mean (SD) & $56.8 \quad(9.7)$ & $54.7 \quad(10.7)$ & & & \\
\hline Region & & & Percutaneous intervention & 0.3 & 0.2 \\
\hline West & 10.8 & 9.4 & Combined comorbidity, mean (SD) & $0.0 \quad(1.0)$ & \multirow{2}{*}{$0.0 \quad(1.0)$} \\
\hline South & 39.4 & 43.2 & Discharged within last 7 days & 0.2 & \\
\hline Midwest & 5.3 & 4.9 & \multicolumn{2}{|l|}{ Health resource utilization } & 0.2 \\
\hline Northeast & 38.8 & 36.1 & All-cause ER visit, mean (SD) & $(0.6)$ & $(0.6)$ \\
\hline Median household income & \multirow{2}{*}{$73,818(25,871)$} & \multirow{2}{*}{$72,291(25,649)$} & Office visits, mean (SD) & $5.3 \quad(6.9)$ & $(6.9)$ \\
\hline$\$$, mean (SD) & & & All-cause hospital days, mean (SD) & $0.3 \quad(2.7)$ & $0.2 \quad(2.1)$ \\
\hline Black, mean (SD) & $12.9 \quad(18.7)$ & $16.9 \quad(23.1)$ & Flu shot & 11.9 & 9.1 \\
\hline High school graduate, mean (SD) & $89.4 \quad(7.0)$ & $88.8 \quad(7.5)$ & Mammogram/PSA & 4.4 & 3.8 \\
\hline \multicolumn{3}{|l|}{ Clinical } & Total copayments, \$, mean (SD) & $196.7(344.4)$ & $152.2(253.6)$ \\
\hline Acute myocardial infarction & 0.5 & 0.3 & Number unique drugs, mean (SD) & $3.8 \quad(3.7)$ & $3.4 \quad(3.5)$ \\
\hline Angina & 0.2 & 0.2 & Filled at $\geq 2$ pharmacies & 29.2 & 26.4 \\
\hline Atrial fibrillation & 0.4 & 0.2 & $\geq 2$ previous 90 -day fills & 18.9 & 20.1 \\
\hline Coronary artery disease & 1.2 & 0.8 & Number filling ZIP codes, mean (SD) & $1.4 \quad(0.8)$ & $1.3 \quad(0.8)$ \\
\hline Cancer & 3.1 & 2.4 & $\begin{array}{l}\text { Number unique days drugs filled, } \\
\text { mean (SD) }\end{array}$ & $6.4 \quad(5.7)$ & $(5.1)$ \\
\hline Chest pain & 5.9 & 6.5 & Index medication & & \\
\hline Congestive heart failure & 0.4 & 0.2 & Combination medication & 11.6 & 11.0 \\
\hline Chronic kidney disease & 2.8 & 2.3 & Brand name medication & 13.8 & 17.2 \\
\hline COPD/asthma & 6.1 & 5.8 & Formulary medication & 91.6 & 91.9 \\
\hline Dementia & 0.3 & 0.3 & Copayment, \$, mean (SD) & $30.1 \quad(42.4)$ & $33.9 \quad(53.7)$ \\
\hline Depression & 5.0 & 4.6 & Mail order use & 30.9 & 24.0 \\
\hline Diabetes & 20.4 & 19.0 & Medication use and adherence & & \\
\hline Hypertension & 47.4 & 40.5 & Number chronic meds filled, & $(1.4)$ & (1.4) \\
\hline Hyperlipidemia & 45.2 & 42.0 & mean (SD) & & \\
\hline Liver disease & 1.6 & 1.5 & Chronic medication adherence, & $(16.3)$ & $(19.4)$ \\
\hline Osteoporosis & 4.0 & 3.9 & mean (SD) & & \\
\hline
\end{tabular}

a Fully Adherent $(P D C \geq 0.8)$ and Poorly Adherent $(P D C<0.8)$ defined in days 1-365 after the date of initiation.

$C O P D=$ chronic obstructive pulmonary disease; $E R=$ emergency room; $P S A=$ prostate specific antigen test; $S D=$ standard deviation; TIA = transient ischemic attack

is considered to more accurately measure adherence than other methods. ${ }^{14-17}$

From the supply diary, we calculated long-term adherence using the proportion of days covered (PDC) measure by dividing the number of days with medication available by the number of days during the 365-day follow-up period (days 1-365). ${ }^{18}$ We also calculated adherence in days 31-365 and days 121-365 after the index date as "delayed" adherence outcome assessment periods for secondary analyses. These 2 measures excluded the first 30 and 120 days of adherence information because, as explained in subsequent sections, variables from these periods were included as predictors in some models. For all adherence outcome measures, we defined "full adherence" as $P D C \geq 0.8$, the cut point used in many quality measures and in previous literature. ${ }^{19,20}$

\section{Predictors}

We measured potential predictors of adherence at baseline and in the first 30-120 days after the index date. The rationale for the different predictor measurement periods was to both identify the optimal set of adherence predictors for this population and also to establish when after initiation that long-term, 12-month adherence could be strongly predicted. All of these variables and their definitions are provided in Appendix A (available in online article).

Baseline. We first defined 50 baseline demographic, clinical, health resource utilization, and medication use variables using enrollment files and medical and pharmacy claims data before and including the index date to identify the predictive ability of baseline characteristics in this population (Table 1). These variables were based on characteristics that have been associated 


\section{Predicting Adherence to Chronic Disease Medications in Patients with Long-term Initial Medication Fills Using Indicators of Clinical Events and Health Behaviors}

\section{TABLE 2 Potential Predictors of Adherence Measured Shortly After Medication Initiation}

\begin{tabular}{|c|c|c|c|c|}
\hline \multirow[b]{2}{*}{ Predictor, \% } & \multicolumn{2}{|c|}{$\begin{array}{l}\text { Fully Adherent }{ }^{\mathrm{a}} \\
\mathrm{n}=12,068\end{array}$} & \multicolumn{2}{|c|}{$\begin{array}{l}\text { Poorly Adherent }{ }^{\mathrm{a}} \\
\mathrm{n}=20,181\end{array}$} \\
\hline & $\begin{array}{l}\text { Days 1-30 After } \\
\text { Initiation }\end{array}$ & $\begin{array}{l}\text { Days } 1-120 \text { After } \\
\text { Initiation }\end{array}$ & $\begin{array}{l}\text { Days 1-30 After } \\
\text { Initiation }\end{array}$ & $\begin{array}{l}\text { Days 1-120 After } \\
\text { Initiation }\end{array}$ \\
\hline \multicolumn{5}{|l|}{ Clinical (new diagnosis) } \\
\hline Acute myocardial infarction & 0.1 & 0.2 & 0.1 & 0.1 \\
\hline Angina & 0.1 & 0.1 & 0.1 & 0.2 \\
\hline Atrial fibrillation & 0.1 & 0.2 & 0.1 & 0.2 \\
\hline Coronary artery disease & 0.1 & 0.5 & 0.1 & 0.4 \\
\hline Cancer & 0.3 & 1.0 & 0.2 & 0.9 \\
\hline Chest pain & 0.8 & 2.4 & 0.8 & 1.9 \\
\hline Congestive heart failure & 0.1 & 0.1 & 0.1 & 0.1 \\
\hline Chronic kidney disease & 0.2 & 0.7 & 0.2 & 0.6 \\
\hline COPD/asthma & 0.4 & 1.4 & 0.4 & 1.3 \\
\hline Dementia & 0.1 & 0.1 & 0.1 & 0.2 \\
\hline Depression & 0.3 & 1.2 & 0.2 & 1.0 \\
\hline Diabetes & 0.8 & 2.7 & 0.6 & 1.7 \\
\hline Hypertension & 2.0 & 5.2 & 1.3 & 3.5 \\
\hline Hyperlipidemia & 1.8 & 5.4 & 1.2 & 3.5 \\
\hline Liver disease & 0.2 & 0.7 & 0.2 & 0.7 \\
\hline Osteoporosis & 0.4 & 1.1 & 0.4 & 1.1 \\
\hline Peripheral vascular disease & 0.1 & 0.2 & 0.1 & 0.2 \\
\hline Stroke/TIA & 0.1 & 0.2 & 0.1 & 0.1 \\
\hline Coronary artery bypass graft surgery & 0.1 & 0.1 & 0.1 & 0.1 \\
\hline Percutaneous intervention & 0.1 & 0.2 & 0.1 & 0.1 \\
\hline Allergic reaction or adverse drug event & 1.6 & 3.1 & 1.7 & 3.2 \\
\hline \multicolumn{5}{|l|}{ Health resource utilization } \\
\hline All-cause ER visit & \multicolumn{2}{|l|}{1.7} & 2.3 & 7.3 \\
\hline Number office visits, mean (SD) & $1.0 \quad(1.7)$ & $3.4 \quad(5.3)$ & $0.9 \quad(1.6)$ & $3.1 \quad(5.3)$ \\
\hline All-cause hospital days & 0.7 & 2.7 & 0.8 & 2.8 \\
\hline Flu shot & 1.1 & 5.6 & 0.9 & 4.3 \\
\hline Mammogram/PSA & 0.6 & 2.3 & 0.6 & 2.0 \\
\hline Total copayments, \$, mean (SD) & \multicolumn{2}{|r|}{$202.3(282.7)$} & $33.3(98.0)$ & $158.3(234.1)$ \\
\hline Number unique drugs, mean (SD) & $1.4 \quad(1.8)$ & $4.9 \quad(3.5)$ & $1.1 \quad(1.6)$ & $3.5 \quad(3.2)$ \\
\hline Filled at $\geq 2$ pharmacies & 9.3 & 35.1 & 6.7 & 25.9 \\
\hline Number filling ZIP codes, mean (SD) & $0.6 \quad(0.7)$ & $1.4 \quad(0.9)$ & $0.5 \quad(0.6)$ & $1.1 \quad(0.8)$ \\
\hline Number unique days drugs filled, mean (SD) & $1.0 \quad(1.3)$ & $5.0 \quad(4.1)$ & $0.9 \quad(1.2)$ & $3.6 \quad(3.7)$ \\
\hline Visit to same provider & 1.4 & 4.2 & 1.5 & 4.1 \\
\hline Visit to same pharmacy & 39.5 & 86.4 & 33.8 & 67.6 \\
\hline Medication use and adherence & & & & \\
\hline Number chronic meds filled, mean (SD) & $0.5 \quad(0.9)$ & $3.5 \quad(3.1)$ & $0.4 \quad(0.7)$ & $2.0 \quad(2.7)$ \\
\hline Refilled prior chronic medication, mean (SD) & $0.6 \quad(0.8)$ & $1.7 \quad(1.1)$ & $0.4 \quad(0.7)$ & $1.4 \quad(1.1)$ \\
\hline Refilled initiated study medication & - & 91.4 & - & 50.8 \\
\hline
\end{tabular}

with adherence in the peer-reviewed literature. Demographic information included age, gender, region, and community-level variables based on the patient's ZIP code of residence. Clinical characteristics were measured using inpatient and outpatient medical claims data and included specific comorbidities such as acute myocardial infarction, congestive heart failure, depression, and stroke, and a combined comorbidity score. ${ }^{21}$
We also measured health resource utilization characteristics like number of all-cause hospitalization days, number of office visits for any indication, pharmacy visits (defined by filling any medication), healthy behaviors (e.g., receipt of flu shot or health screening), geographic spread in pharmacy visits (e.g., number of distinct ZIP codes in which any medications were filled at pharmacies), medication synchronization (e.g., number of distinct days in which any medications were filled), ${ }^{22}$ and 


\section{Predicting Adherence to Chronic Disease Medications in Patients with Long-term Initial Medication Fills Using Indicators of Clinical Events and Health Behaviors}

number of all-cause ER visits. Medication use predictors were measured using pharmacy claims and included characteristics of the index medication, such as copayments, brand name status, formulary status, and mail order use, as well as prior chronic medication use and adherence. Each characteristic was separately measured in the 180 days before the index date.

Thirty Days After Initiation. We measured the new occurrence of the same clinical and health resource utilization variables in the first 30 days after the index date as during the 180-day baseline period (Appendix A). We hypothesized that these short-term characteristics and behaviors might be associated with subsequent adherence and could be measured rapidly in individuals after initiation. If successful, these predictors could be used in rapidly deployed adherence interventions. We defined clinical events or diagnoses as "new" if they had not previously occurred in administrative claims during the patient's entire baseline period of eligibility. Health resource utilization variables and other medication use variables were also remeasured in the first 30 days after the index date, including whether the patient refilled a previously filled chronic medication. In addition, as potential indicators of continuity of care, we measured whether patients returned for any reason to the same provider or pharmacy in which they were prescribed or filled their initial medication (Table 2).

One Hundred Twenty Days After Initiation. We then measured variables within the first 120 days after the index date. Previous research has found that indicator variables of shortterm refilling within the first 30-90 days were strong predictors of future adherence among patients with shorter supplies, so we hypothesized that extending these methods would improve adherence prediction among patients filling longer supplies. By definition, patients initiating 90-day supplies are fully adherent for the first 90 days of therapy; therefore, these direct measures of adherence could not be directly applied in settings of longer medication fills.

Therefore, we repeated the measurements of the new clinical diagnoses, acute clinical events, and health resource utilization variables as done in the 30 days after initiation but also added an indicator of refilling the initiated medication during the 120 days after the index date (Appendix A). Because by definition all patients would have the same level of adherence in the first 90 days (days 1-90), we also measured whether the patient was optimally adherent (PDC $\geq 0.8$ ) during the fourth 30-day period (days 91-120) after the index date, as a postinitiation adherence indicator. ${ }^{5,6}$ In other words, patients would be optimally adherent in this fourth 30-day period if they refilled no later than day 96 and received $\geq 24$ days supply in that refill.

\section{Statistical Analysis}

First, we examined mean PDC and full adherence for each of the 3 adherence measures for the 3 disease states. Then, for each of the 3 adherence outcome measures, we estimated
5 main prediction models of adherence in the full cohort and in strata by disease state using logistic regression. Model 1 used only baseline predictors. Models 2 and 3 were estimated using only the variables in the first 30 days and 120 days after initiation, respectively. Model 4 included baseline predictors and the variables in the first 120 days after initiation. Model 5 included all of these sets of predictors, including variables in the first 30 and 120 days after initiation. We also reestimated these models within subgroups by chronic disease (e.g., hypertension, diabetes, hyperlipidemia) to see whether there were any differences in prediction. Additional combinations of predictors were estimated in secondary models.

Using the c-statistic, we evaluated the ability of models to predict adherence based on their ability to distinguish between patients who did and did not become adherent. ${ }^{23}$ This measure ranges from 0.5 (noninformative) to 1.0 (perfect prediction). ${ }^{24,25}$ Models with higher c-statistics are generally better at identifying patients who are likely to experience the outcome and thus are potentially more useful for future interventions. For all models, we performed 10-fold cross-validation to avoid bias associated with evaluating predictions on the same data that are used to estimate the model. ${ }^{26}$

\section{Results}

Of the 425,743 patients who filled a study medication during the study period, 198,766 patients were new initiators. Of the total new initiators, 32,249 (16.2\%) had dispensations of at least 90 days, including 14,930 statin, 4,432 oral hypoglycemic, and 12,887 antihypertensive initiators. Baseline characteristics of the study cohort by adherence are shown in Table 1. Patients' mean age was 55.5 years (standard deviation $[S D]=10.4$ ), and $43.3 \%$ were female.

In total, $39.4 \%$ of patients were adherent in the 12 months after treatment initiation (mean PDC $[S D]=0.68[0.27]$ ). Adherence was similar across all 3 disease states; $37.9 \%$, $35.5 \%$, and $43.6 \%$ were adherent to statins, oral hypoglycemics, and antihypertensives, respectively. Rates of adherence were slightly lower when adherence was assessed over the shorter outcome assessment windows; for instance, 37.4\% of patients were adherent within the 121-365-day window compared with $39.4 \%$ for the 1-365-day window.

Table 2 shows the rates of the new clinical diagnoses/events, health resource utilization, and medication use characteristics in the 30 and 120 days after initiation that were used in prediction analyses. Of note, $66.0 \%$ of patients refilled the index medication within the first 120 days after initiation. In addition, $68.1 \%$ of patients who were subsequently adherent in days 121-365 were adherent in month 4 (days 91-120), compared with $22.1 \%$ who were subsequently poorly adherent.

The prediction accuracy of the models is shown in Table 3, as quantified by cross-validated c-statistics for all 3 adherence measures. Prediction of 1-year adherence using baseline variables was relatively poor (model 1 cross-validated 


\begin{tabular}{l} 
TABLE 3 $\begin{array}{l}\text { Cross-Validated C-statistics from Models for Adherence Among Patients Filling Long-term } \\
\text { Medication Supplies }\end{array}$ \\
\cline { 2 - 4 } \\
\cline { 2 - 4 } Model
\end{tabular}

c-statistic $=0.644)$. Prediction was worse using only predictors from the first 30 days after initiation (model $2 \mathrm{c}=0.545$ ). Clinical, health resource utilization, and medication use and adherence predictors in the first 120 days after initiation substantially improved predictive ability (model $3 \mathrm{c}=0.823$ ). The model that incorporated all baseline characteristics and predictors within the first 120 days after medication initiation strongly predicted future adherence (model $4 \mathrm{c}=0.832$ ). The best-performing model incorporated all of the predictors (model $5 \mathrm{c}=0.837$ ). The multivariable contributions of each predictor measured shortly after medication initiation (from models 2 and 3) are shown in Table 4, suggesting that, for example, pharmacy-specific health behaviors (such as visiting the same pharmacy and number of unique filling ZIP codes) are associated with optimal adherence.

Additional combinations of predictors were also evaluated, and their results are also presented in Table 3. Of note, a model estimated using only the indicator of refilling the medication in the first 120 days yielded a cross-validated c-statistic of 0.714. The indicator of optimal adherence in month 4 was the strongest individual predictor of adherence, even among the delayed 31-365-day and 121-365-day adherence outcome measures $(c=0.744)$.

Overall, the ability to predict adherence was similar for all 3 adherence outcome assessment windows. For instance, the bestperforming model (model 5) was still strongly predictive of adherence over days 31-365 ( $c=0.842)$ and days 121-365 ( $c=0.818)$ compared with the primary outcome of days $1-365(\mathrm{c}=0.837)$. Using the delayed adherence outcome assessment windows did not affect the ability to predict subsequent adherence.
Appendix B (available in online article) presents the crossvalidated c-statistics of the predictive ability of adherence for each of the 3 chronic disease conditions. The ability to predict adherence was similar across all 3 disease areas for the different models.

\section{Discussion}

Using a large cohort of patients initiating chronic disease medications, we evaluated whether short-term filling behaviors and novel predictors after initiation improved the ability to predict adherence among patients receiving longer prescription dispensations. An approach using indicators of adherence and refilling shortly after initiation was highly accurate and had much better predictive ability compared with baseline information alone. Predictors including new clinical events and health resource utilization within the first 120 days after initiation also strongly predicted adherence. Overall, we found that a combination of clinical, health resource utilization, and medication filling characteristics before and after treatment initiation led to highly accurate predictions of adherence.

Efforts to predict long-term adherence to medications have mainly focused on patients who fill short initial supplies, with varying degrees of success..$^{5-8}$ While rates of adherence are generally higher among patients who fill longer initial supplies (e.g., 90-day supplies) than shorter initial supplies (e.g., 30-day supplies), prediction has been more difficult for these patients. ${ }^{5,6}$ These results confirm previous research that has found that predictions of adherence using only baseline information from administrative claims databases are relatively poor. ${ }^{5,6,27,28}$ As seen in models examining subsets of baseline characteristics, none of the categories of baseline characteristics, including demographic, clinical, health resource 


\section{Predicting Adherence to Chronic Disease Medications in Patients with Long-term Initial Medication Fills Using Indicators of Clinical Events and Health Behaviors}

\begin{tabular}{|c|c|c|}
\hline \multirow[b]{2}{*}{ Predictor of Full Adherence } & & \\
\hline & $\begin{array}{l}\text { Model } 2 \text { : Variables } \\
\text { from Days 1-30 } \\
\text { After Initiation }\end{array}$ & $\begin{array}{c}\text { Model 3: Variables } \\
\text { from Days 1-120 } \\
\text { After Initiation }\end{array}$ \\
\hline Intercept & $-0.571 \quad(0.017)$ & $-1.690(0.033)$ \\
\hline \multicolumn{3}{|l|}{ Clinical (new diagnosis) } \\
\hline Acute myocardial infarction & $0.857(0.874)$ & $0.458(0.478)$ \\
\hline Angina & $1.241(0.825)$ & $0.760(0.520)$ \\
\hline Atrial fibrillation & $1.320(0.733)$ & $0.874(0.416)$ \\
\hline Coronary artery disease & $-0.305(0.583)$ & $-0.114(0.303)$ \\
\hline Cancer & $0.275(0.239)$ & $0.081(0.138)$ \\
\hline Chest pain & $-0.989(0.144)$ & $0.092(0.090)$ \\
\hline Congestive heart failure & $0.484(0.934)$ & $-1.517(0.500)$ \\
\hline Chronic kidney disease & $0.017(0.289)$ & $-0.183(0.171)$ \\
\hline COPD/asthma & $-0.155(0.208)$ & $-0.201 \quad(0.117)$ \\
\hline Dementia & $0.003(0.594)$ & $-0.028(0.333)$ \\
\hline Depression & $0.147(0.230)$ & $-0.108(0.125)$ \\
\hline Diabetes & $0.146(0.149)$ & $-0.076(0.095)$ \\
\hline Hypertension & $0.333(0.102)$ & $0.026(0.067)$ \\
\hline Hyperlipidemia & $0.371(0.109)$ & $0.253(0.066)$ \\
\hline Liver disease & $0.056(0.255)$ & $-0.109(0.157)$ \\
\hline Osteoporosis & $0.075(0.201)$ & $-0.061(0.125)$ \\
\hline Peripheral vascular disease & $0.349(0.452)$ & $-0.174(0.318)$ \\
\hline Stroke/TIA & $-0.096(0.787)$ & $0.091 \quad(0.421)$ \\
\hline $\begin{array}{l}\text { Coronary artery bypass graft } \\
\text { surgery }\end{array}$ & $1.156(0.952)$ & $0.547(0.562)$ \\
\hline Percutaneous intervention & $0.767(0.510)$ & $0.406(0.397)$ \\
\hline $\begin{array}{l}\text { Allergic reaction or adverse } \\
\text { drug event }\end{array}$ & $-0.497(0.336)$ & $-0.445 \quad(0.170)$ \\
\hline \multicolumn{3}{|l|}{ Health resource utilization } \\
\hline All-cause ER visit & $-0.445(0.095)$ & $-0.418(0.058)$ \\
\hline Number office visits & $-0.006(0.008)$ & $-0.017(0.003)$ \\
\hline All-cause hospital days & $-0.261(0.171)$ & $-0.278(0.097)$ \\
\hline Flu shot & $0.222(0.119)$ & $0.086(0.059)$ \\
\hline Mammogram/PSA & $-0.185(0.157)$ & $0.038(0.089)$ \\
\hline Total copayments & $-0.001(0.001)$ & $-0.001(0.001)$ \\
\hline Number unique drugs & $-0.002(0.018)$ & $0.113(0.008)$ \\
\hline Filled at $\geq 2$ pharmacies & $-0.039(0.132)$ & $-0.272(0.062)$ \\
\hline Number filling ZIP codes & $0.157(0.134)$ & $0.358(0.065)$ \\
\hline $\begin{array}{l}\text { Number unique days drugs } \\
\text { filled }\end{array}$ & $0.052(0.025)$ & $-0.044(0.006)$ \\
\hline Visit to same provider & $-0.166(0.104)$ & $-0.101(0.068)$ \\
\hline Visit to same pharmacy & $-0.013(0.036)$ & $0.542(0.040)$ \\
\hline \multicolumn{3}{|l|}{ Medication use and adherence } \\
\hline Number chronic meds filled & $0.032(0.023)$ & $0.109(0.007)$ \\
\hline $\begin{array}{l}\text { Refilled prior chronic } \\
\text { medication }\end{array}$ & $0.076(0.030)$ & $-0.083(0.017)$ \\
\hline $\begin{array}{l}\text { Refilled initiated study } \\
\text { medication }\end{array}$ & - & $2.259(0.040)$ \\
\hline
\end{tabular}

${ }^{a}$ Model predicting adherence in days 1-365 after the date of medication initiation. $C O P D=$ chronic obstructive pulmonary disease; $E R=$ emergency room; $P S A=$ prostate specific antigen test; $S E=$ standard error; TIA = transient ischemic attack. utilization, or factors associated with the index medication like copayment or mail order use, added meaningfully to the prediction of long-term adherence. One explanation could be that patients' medication-taking behaviors are shaped more by contemporary factors that happen while starting the medication rather than factors before initiating the medication.

One successful previous approach to adherence prediction in other settings involves including simple indicators of filling within the first 90 days after medication initiation, reaching cross-validated c-statistics of 0.827 among patients who initiated 30-day supplies. ${ }^{5,6}$ However, even with these short-term patterns of medication filling, the prediction of long-term adherence has been extremely poor for patients initiating longer term supplies (e.g., cross-validated c-statistic $=0.591$ ) because, by definition, patients are classified as adherent for the first 90 days. ${ }^{6}$ Prior work has also suggested that patients initiating longer initial supplies are slightly healthier than patients initiating 30-day supplies. ${ }^{6}$ Therefore, we sought to develop methods to improve the prediction of long-term adherence among patients filling longer initial medication supplies, as these patients may have different underlying behaviors and be more medically stable than other better-understood patient populations.

Our study extends previous studies by empirically evaluating whether a combination of acute clinical events, health resource utilization, and medication refill patterns after initiation predict long-term adherence. In this study, prediction using only information from the first 30 days after initiating the medication was relatively ineffective. One explanation could be the low prevalence of these indicators; predictors with low prevalence do not substantially increase predictive ability. Another explanation could be that a clinical event within the first 30 days could be too early relative to the need to refill the medication to aid in prediction.

By contrast, extending indicators of acute clinical events, health resource utilization, and medication fill patterns within the first 120 days after initiation were strongly predictive of future adherence across all 3 adherence outcome assessment windows, much more than all measured baseline characteristics combined. In particular, when examining the suite of potential behavioral factors measured shortly after initiation, the indicators of refilling and adherence shortly after filling appeared to be the strongest predictors of adherence, but the other behavioral factors also meaningfully contributed to adherence prediction.

Overall, our findings support the conclusion that using any of these characteristics or behaviors measured in the 120 days shortly after initiation, but particularly a simple indicator of whether the initial medication was refilled or adhered to, can substantially improve adherence predictions. However, some of these other health behaviors or clinical events, such as returning to the same pharmacy, changes in clinical status, and increasing health resource utilization, appeared to have some utility in model prediction. 


\section{Predicting Adherence to Chronic Disease Medications in Patients with Long-term Initial Medication Fills Using Indicators of Clinical Events and Health Behaviors}

In an effort to address the problem of medication adherence, particularly in chronic conditions such as hyperlipidemia, hypertension, and diabetes, ${ }^{2,29}$ payers have been increasing the allowed number of dispensable pills per prescription. This practice, which provides patients with more pills per prescription so that fewer refill requests are necessary, is becoming increasingly common. ${ }^{10,11}$ In our study, the number of patients who initiated 90-day supplies was quite substantial, exceeding $15 \%$ of the total number of patients starting therapy. This number is similar to rates from our previous research and is a trend that is increasing in the industry. ${ }^{6,11}$ In response to this trend, our approach to predicting adherence among these patients might also provide some insight into the types of patients who are mostly likely to adhere to longer medication dispensations.

While many clinical and behavioral interventions with the aim of improving adherence have been studied, relatively few have been successful. ${ }^{30}$ One reason why nonadherence remains a problem, despite intervention, is that adherence is a complex behavior that can change over time. Even when we can intervene, interventions may not be optimally timed. . $^{7,31}$

The results from this study may help inform the development of interventions by providing insight into when adherence interventions may be effectively measured and deployed for this population. In general, patients' actions within the first few months after initiation appear to be crucial for determining whether these patients will continue to be adherent. Health plans wishing to identify members using long-term medication supplies at risk of nonadherence may be able to use these health behavior and refilling indicators to identify appropriate patients around their 120th day of treatment for potential intervention. While these patients may be identified slightly later than those with shorter initial dispensations, it may still be early enough to affect overall 1-year adherence, though not all patients may respond to this type of intervention.

\section{Limitations}

The findings of this study should be interpreted in light of several limitations. First, like other studies, medication adherence was measured indirectly in patients using days supply, a validated method that has been shown to correlate well with electronic records and patient self-report. ${ }^{32}$ However, there could still be potential for misclassification in adherence, particularly among patients whose providers discontinued or substantially modified their treatment. It is also possible that some of the patients included in our study of new initiators may have previously filled the medication; as in other studies, we excluded patients who recently filled the medication., ${ }^{5,6}$ Moreover, if the patient had not used the medication in the past 6 months, then response and willingness to adhere to therapy would likely be similar to those who more recently initiated therapy.
We also included patients with an initial supply of $>90$ days; prediction accuracy may have improved if excluding patients filling initial supplies of $\geq 120$ days. Overall prediction accuracy is also evaluated at the study population level; there is heterogeneity within the patient sample, so individual patients may have different factors associated with their long-term adherence.

In addition, the results of this study also cannot be fully generalized to other payer systems, such as Medicare or Medicaid, or to patients initiating shorter initial supplies of medications (e.g., $\leq 30$ days), as the study populations and factors associated with adherence and behaviors could differ. Finally, there is a potential downside to starting patient intervention closer to 90 or 120 days after treatment initiation, since discontinuation of the medication could have occurred by then. However, few effective adherence support interventions begin at the time of medication initiation, and use of such targeted interventions with demonstrably nonadherent patients is more effective than use of nontargeted interventions. ${ }^{33}$

\section{Conclusions}

The results of this study provide an accurate way to predict adherence among the growing subgroup of patients filling longer supplies of medication, using a combination of clinical, health resource utilization, and medication filling characteristics before and after treatment initiation. Efforts to predict adherence among these patients for potential intervention may benefit from examining and including these predictors.

\section{Authors}

JULIE C. LAUFFENBURGER, PharmD, PhD; ALEXIS A. KRUMME, ScD; and NITEESH K. CHOUDHRY, MD, PhD, Division of Pharmacoepidemiology and Pharmacoeconomics and Center for Healthcare Delivery Sciences, Department of Medicine, Brigham and Women's Hospital and Harvard Medical School, Boston, Massachusetts. JESSICA M. FRANKLIN, PhD, and GREGORY BRILL, MS, Division of Pharmacoepidemiology and Pharmacoeconomics, Department of Medicine, Brigham and Women's Hospital and Harvard Medical School, Boston, Massachusetts. WILLIAM H. SHRANK, MD, MSHS, and OLGA S. MATLIN, PhD, CVS Health, Woonsocket Rhode Island. CLAIRE M. SPETTELL, PhD, Data Science, Aetna, Hartford, Connecticut.

AUTHOR CORRESPONDENCE: Niteesh K. Choudhry, MD, PhD, Brigham and Women's Hospital, Harvard Medical School, 1620 Tremont St., Ste. 3030, Boston, MA 02120. Tel.: 617.278.0930; E-mail:nkchoudhry@bwh.harvard.edu. 


\section{Predicting Adherence to Chronic Disease Medications in Patients with Long-term Initial Medication Fills Using Indicators of Clinical Events and Health Behaviors}

\section{DISCLOSURES}

This work was supported by an unrestricted grant from CVS Health to Brigham and Women's Hospital. Shrank and Matlin were employees and shareholders at CVS Health at the time of this study; they report no financial interests in products or services that are related to this subject. Spettell is an employee of, and shareholder in, Aetna.

This research was previously presented at the 2016 Annual Conference of the International Society for Pharmacoepidemiology; August 25-28, 2016; Dublin, Ireland.

\section{REFERENCES}

1. Ho PM, Bryson CL, Rumsfeld JS. Medication adherence: its importance in cardiovascular outcomes. Circulation. 2009:119(23):3028-35.

2. Wang B, Choudhry NK, Gagne JJ, Landon J, Kesselheim AS. Availability and utilization of cardiovascular fixed-dose combination drugs in the United States. Am Heart J. 2015;169(3):379-86.el.

3. Iuga AO, McGuire MJ. Adherence and health care costs. Risk Manag Healthe Policy. 2014;7:35-44.

4. Sokol MC, McGuigan KA, Verbrugge RR, Epstein RS. Impact of medication adherence on hospitalization risk and healthcare cost. Med Care. 2005:43(6):521-30.

5. Franklin JM, Krumme AA, Shrank WH, Matlin OS, Brennan TA, Choudhry NK. Predicting adherence trajectory using initial patterns of medication filling. Am J Manag Care. 2015;21(9):e537-44.

6. Franklin JM, Shrank WH, Lii J, et al. Observing versus predicting: initial patterns of filling predict long-term adherence more accurately than highdimensional modeling techniques. Health Serv Res. 2016;51(1):220-39.

7. Franklin JM, Shrank WH, Pakes J, et al. Group-based trajectory models: a new approach to classifying and predicting long-term medication adherence. Med Care. 2013;51(9):789-96.

8. Zimolzak AJ, Spettell CM, Fernandes J, et al. Early detection of poor adherers to statins: applying individualized surveillance to pay for performance. PLoS One. 2013;8(11):e79611.

9. Krumme AA, Franklin JM, Isaman DL, et al. Predicting l-year statin adherence among prevalent users: a retrospective cohort study. J Manag Care Spec Pharm. 2017;23(4):494-502. Available at: https://www.jmcp.org/ doi/10.18553/jmcp.2017.23.4.494.

10. Khandelwal N, Duncan I, Rubinstein E, et al. Medication adherence for 90-day quantities of medication dispensed through retail and mail order pharmacies. Am J Manag Care. 2011;17(11):e427-34.

11. Liberman JN, Girdish C. Recent trends in the dispensing of 90-daysupply prescriptions at retail pharmacies: implications for improved convenience and access. Am Health Drug Benefits. 2011;4(2):95-100.

12. Taitel M, Fensterheim L, Kirkham H, Sekula R, Duncan I. Medication days' supply, adherence, wastage, and cost among chronic patients in Medicaid. Medicare Medicaid Res Rev. 2012;2(3)

13. National Committee for Quality Assurance. HEDIS 2015 final NDC lists. Available at: http://www.ncqa.org/hedis-quality-measurement/hedismeasures/hedis-2015/hedis-2015-ndc-license/hedis-2015-final-ndc-lists. Accessed April 3, 2018.

14. Martin BC, Wiley-Exley EK, Richards S, Domino ME, Carey TS, Sleath BL. Contrasting measures of adherence with simple drug use, medication switching, and therapeutic duplication. Ann Pharmacother. 2009;43(1):36-44

15. Choudhry NK, Shrank WH, Levin RL, et al. Measuring concurrent adherence to multiple related medications. Am J Manag Care. 2009;15(7):457-64
16. Yeaw J, Benner JS, Walt JG, Sian S, Smith DB. Comparing adherence and persistence across 6 chronic medication classes. J Manag Care Pharm. 2009;15(9):728-40. Available at: https://www.jmcp.org/doi/10.18553/ jmcp.2009.15.9.728.

17. Amin K, Farley JF, Maciejewski ML, Domino ME. Effect of Medicaid policy changes on medication adherence: differences by baseline adherence. J Manag Care Spec Pharm. 2017;23(3):337-45. Available at: https://www.jmcp. org/doi/full/10.18553/jmcp.2017.23.3.337.

18. Benner JS, Glynn RJ, Mogun H, Neumann PJ, Weinstein MC, Avorn J Long-term persistence in use of statin therapy in elderly patients. JAMA. 2002;288(4):455-61.

19. Centers for Medicare \& Medicaid Services. Medicare health \& drug plan quality and performance ratings: 2013 Part C \& Part D technical notes. In: 2013 plan ratings and display measures. Available at: https://www.cms. gov/Medicare/Prescription-Drug-Coverage/PrescriptionDrugCovGenIn/ PerformanceData.html. Accessed April 3, 2018.

20. National Committee for Quality Assurance. 2014 HEDIS summary table of measures, product lines and changes. Available at: http://www.ncqa. org/Portals/O/HEDISQM/HEDIS2014/List\%20of\%20HEDIS\%202014\%20 Measures.pdf. Accessed April 3, 2018

21. Gupta AK, Arshad S, Poulter NR. Compliance, safety, and effectiveness of fixed-dose combinations of antihypertensive agents: a meta-analysis. Hypertension. 2010;55(2):399-407.

22. Holdford DA, Inocencio TJ. Adherence and persistence associated with an appointment-based medication synchronization program. J Am Pharm Assoc. 2013;53(6):576-83

23. Efron B, Gong G. A leisurely look at the bootstrap, the jackknife, and cross-validation. Am Stat. 1983;37(1):36-48.

24. Harrell FE Jr, Lee KL, Mark DB. Multivariable prognostic models: issues in developing models, evaluating assumptions and adequacy, and measuring and reducing errors. Stat Med. 1996;15(4):361-87.

25. Cook NR. Clinically relevant measures of fit? A note of caution. Am J Epidemiol. 2012;176(6):488-91.

26. Steyerberg EW, Harrell FE Jr, Borsboom GJ, Eijkemans MJ, Vergouwe Y, Habbema JD. Internal validation of predictive models: efficiency of some procedures for logistic regression analysis. J Clin Epidemiol. 2001;54(8):774-81.

27. Pan F, Chernew ME, Fendrick AM. Impact of fixed-dose combination drugs on adherence to prescription medications. J Gen Intern Med. 2008; 23(5):611-14.

28. Gu Q, Burt VL, Dillon CF, Yoon S. Trends in antihypertensive medication use and blood pressure control among United States adults with hypertension: the National Health And Nutrition Examination Survey, 2001 to 2010. Circulation. 2012;126(17):2105-14

29. Choudhry NK, Glynn RJ, Avorn J, et al. Untangling the relationship between medication adherence and post-myocardial infarction outcomes: medication adherence and clinical outcomes. Am Heart J. 2014;167(1):51-58.e5.

30. Viswanathan M, Golin CE, Jones CD, et al. Interventions to improve adherence to self-administered medications for chronic diseases in the United States: a systematic review. Ann Intern Med. 2012;157(11):785-95.

31. Nieuwlaat R, Wilczynski N, Navarro T, et al. Interventions for enhancing medication adherence. Cochrane Database Syst Rev. 2014:11:CD000011.

32. Hansen RA, Kim MM, Song L, Tu W, Wu J, Murray MD. Comparison of methods to assess medication adherence and classify nonadherence. Ann Pharmacother. 2009:43(3):413-22

33. Cutrona SL, Choudhry NK, Fischer MA, et al. Modes of delivery for interventions to improve cardiovascular medication adherence. Am J Manag Care. 2010;16(12):929-42 


\section{Predicting Adherence to Chronic Disease Medications in Patients with Long-term Initial Medication Fills Using Indicators of Clinical Events and Health Behaviors}

\section{APPENDIX A Predictors of Adherence Included in Models}

\section{Type and Timing}

\section{Baseline}

Demographic

\section{Predictors and Relevant ICD-9-CM or CPT-4 Codes}

- Age, gender, region, number of days continuously enrolled in health plan: measured in enrollment file

- Median household income, percentage black, percentage high school graduate: measured at patient's ZIP code level using the linked 2010 U.S. census file

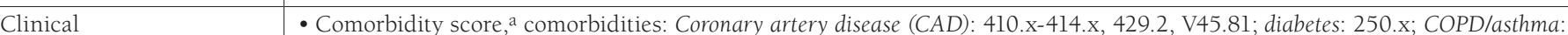
490-493; hypertension: 401.x-405.x, 437.2; hyperlipidemia: 272; congestive heart failure (CHF): 428.x, 398.91, 402.01, 402.11, 402.91, 404.01, 404.11, 404.91, 404.03, 404.13, 404.93; stroke: 433.x1, 434.x1, 435.x, 436, 437.1x, 437.9x, 438.x; depression: 311, 296.2, 296.3, 300.4, 301.12, 309.1; liver disease: 570-573, 456-456.21; kidney disease: 582, 583, 585, 586, 587, 250.4, 250.40, 250.41, 250.42, 250.43, 403.xx, 404.xx, 585.6; dementia: 290.0x-290.9x, 294.1x, 294.9x, 330.x, 331.x; peripheral vascular disease: 433.9, 433.90, and 433.91, 433.30, 433.31, 433.8, 433.80, 433.81; osteoporosis: 733; angina: ICD-9-CM procedure code 36.06 or 36.07 or CPT-4 code 392980, 92981; atrial fibrillation: 427.31 ; chest pain: 786.5 ; cancer: $140 . x-195 . x$, 196.x-198.x, 199.x, 200.x-208.x, 230.x-234.x, 235.x-238.x, 239.x; coronary artery bypass graft (CABG): ICD-9-CM procedure: $36.1 x, 36.2 x$ or CPT-4 code: 33510-33545; percutaneous intervention (PCI): ICD-9-CM procedure code 00.66, 36.01, 36.02, 36.05, 36.06, 36.07, 36.09 or CPT-4 code 92973, 92980, 92981, 92982, 92984, 92995, 92996; allergic reaction: 995.0, 995.2, 995.3, 708.0, 708.9, 695.1, 995.1: measured in medical claims using ICD-9-CM codes unless otherwise specified

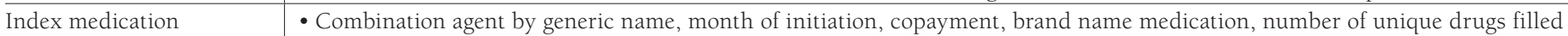
on index date, sum of copayments for all drugs on the index date, formulary medication indicator, mail order use: measured in pharmacy claims

\begin{tabular}{l|l}
\hline Health resource & - Number of ER visits, number of days hospitalized, number of office visits, mammogram/PSA: CPT-4 code: 76082,76083,
\end{tabular} utilization 76092, G0202, G0203, G0103, 84153, 84152-84154; lab test: CPT-4 code: 80061, 83700, 83701, 83704, 83721, 83036,

83037: measured in medical claims using ICD-9-CM codes unless otherwise specified

- Number of unique drugs by generic name, number of unique pharmacies by pharmacy ID, number of filling ZIP codes (distinct ZIP codes that pharmacies were used in), med synchronicity (number of distinct days that medications were filled), number of chronic drugs filled, number of previous 90-day fills: measured in pharmacy claims

- Flu shot: measured in pharmacy or medical claims using procedure codes

Medication use/adherence

- Chronic medication use (defined by filling $\geq 1$ medication from 1 of 25 different chronic medication classes; prior adherence to chronic medications (as measured by proportion of days covered in the baseline period) ${ }^{\mathrm{b}}$ : measured in pharmacy claims

Days 1-30 after initiation

Clinical (new events)c

- CAD: 410.x-414.x, 429.2, V45.81; diabetes: 250.x; COPD/asthma: 490-493; hypertension: 401.x-405.x, 437.2; hyperlipidemia: 272; CHF; 428.x, 398.91, 402.01, 402.11, 402.91, 404.01, 404.11, 404.91, 404.03, 404.13, 404.93; stroke: 433.xl, 434.xl, 435.x, 436, 437.1x, 437.9x, 438.x; depression: 311, 296.2, 296.3, 300.4, 301.12, 309.1; liver disease: 570-573, 456-456.21; kidney disease: 582, 583, 585, 586, 587, 250.4, 250.40, 250.41, 250.42, 250.43, 403.xx, 404.xx, 585.6; dementia: 290.0x-290.9x, 294.1x, 294.9x, 330.x, 331.x; peripheral vascular disease: 433.9, 433.90, and 433.91, 433.30, 433.31, 433.8, 433.80, 433.81; osteoporosis: 733; angina: ICD-9-CM procedure code 36.06 or 36.07 or CPT-4 code 392980, 92981; atrial fibrillation: 427.31; chest pain: 786.5; cancer: 140.x-195.x, 196.x-198.x, 199.x, 200.x-208.x, 230.x-234.x, 235.x-238.x, 239.x; CABG: ICD-9-CM procedure: 36.1x, 36.2x or CPT-4 code: 33510-33545; PCI: ICD-9-CM procedure code 00.66, 36.01, 36.02 36.05, 36.06, 36.07, 36.09 or CPT-4 code 92973, 92980, 92981, 92982, 92984, 92995, 92996; allergic reaction: 995.0, 995.2, 995.3,708.0, 708.9, 695.1, 995.1; measured in medical claims using ICD-9-CM codes unless otherwise specified

Health resource

utilization
- ER visit, number of days hospitalized, number of office visits, mammogram/PSA: CPT-4 code 76082, 76083, 76092, G0202, G0203, G0103, 84153, 84152-84154; lab test: CPT-4 code 80061, 83700, 83701, 83704, 83721, 83036, 83037; visit to same provider as prescribed the initiated medication: measured in medical claims

- Number of unique drugs by generic name, filling at $\geq 2$ distinct pharmacies by pharmacy ID, number of filling ZIP codes (distinct ZIP codes that pharmacies were used in), med synchronicity (number of distinct days that medications were filled), number of chronic drugs filled, number of previous 90-day fills, visit to same pharmacy as where the initiated medication was filled: measured in pharmacy claims

- Flu shot: measured in pharmacy or medical claims using procedure codes to chronic medications (as measured by proportion of days covered in the baseline period)c: measured in pharmacy claims 


\section{APPENDIX A Predictors of Adherence Included in Models (continued)}

Days 1-120 after initiation

Clinical (new events)c

utilization

- CAD: 410.x-414.x, 429.2, V45.81; diabetes: 250.x; COPD/asthma: 490-493; hypertension: 401.x-405.x, 437.2; hyperlipidemia: 272; CHF: 428.x, 398.91, 402.01, 402.11, 402.91, 404.01, 404.11, 404.91, 404.03, 404.13, 404.93; stroke: 433.xl, 434.xl, 435.x, 436, 437.1x, 437.9x, 438.x; depression: 311, 296.2, 296.3, 300.4, 301.12, 309.1; liver disease: 570-573, 456-456.21; kidney disease: 582, 583, 585, 586, 587, 250.4, 250.40, 250.41, 250.42, 250.43, 403.xx, 404.xx, 585.6; dementia: 290.0x-290.9x, 294.1x, 294.9x, 330.x, 331.x; peripheral vascular disease: 433.9, 433.90, and 433.91, 433.30, 433.31, 433.8, 433.80, 433.81; osteoporosis: 733; angina: ICD-9-CM procedure code 36.06 or 36.07 or CPT-4 code 392980, 92981; atrial fibrillation: 427.31; chest pain: 786.5; cancer: 140.x-195.x, 196.x-198.x, 199.x, 200.x-208.x, 230.x-234.x, 235.x-238.x, 239.x; CABG: ICD-9-CM procedure: 36.1x, 36.2x or CPT-4 code: 33510-33545; PCI: ICD-9-CM procedure code 00.66, 36.01, 36.02, 36.05, 36.06, 36.07, 36.09 or CPT-4 code 92973, 92980, 92981, 92982, 92984, 92995, 92996; allergic reaction: 995.0, 995.2, 995.3,708.0, 708.9, 695.1, 995.1: measured in medical claims using ICD-9-CM codes unless otherwise specified

- ER visit, number of days hospitalized, number of office visits, mammogram/PSA: CPT-4 code 76082, 76083, 76092, G0202, G0203, G0103, 84153, 84152-84154; lab test: CPT-4 code 80061, 83700, 83701, 83704, 83721, 83036, 83037; visit to same provider as prescribed the initiated medication: measured in medical claims

- Number of unique drugs by generic name, filling at $\geq 2$ distinct pharmacies by pharmacy ID, number of filling ZIP codes (distinct ZIP codes that pharmacies were used in), med synchronicity (number of distinct days that medications were filled), number of chronic drugs filled, number of previous 90-day fills, visit to same pharmacy as where the initiated medication was filled: measured in pharmacy claims

- Flu shot: measured in pharmacy or medical claims using procedure codes

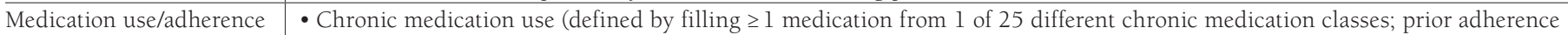
to chronic medications (as measured by proportion of days covered in the baseline period)c; indicator of refilling the study medication: measured in pharmacy claims

Days 91-120 after initiation

Indicator of adherence to $\quad$ - Optimal adherence (defined by PDC $\geq 0.8$ ) in month 4 (days 91-120): measured in pharmacy claims initiated medication

aGagne JJ, et al. J Clin Epidemiol. 2011;64:749-59.

bused as interaction variable with the chronic medication use indicator.

cDefined by not being observed during the patient's entire baseline period of eligibility.

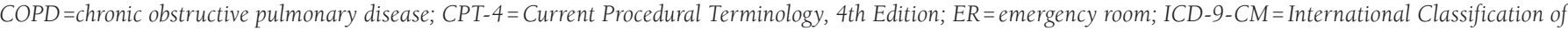
Diseases, Ninth Revision, Clinical Modification; PDC=proportion of days covered; PSA=prostate specific antigen test.

\section{APPENDIX B Cross-Validated C-statistics for Predicting Adherence Among Medication Subgroups}

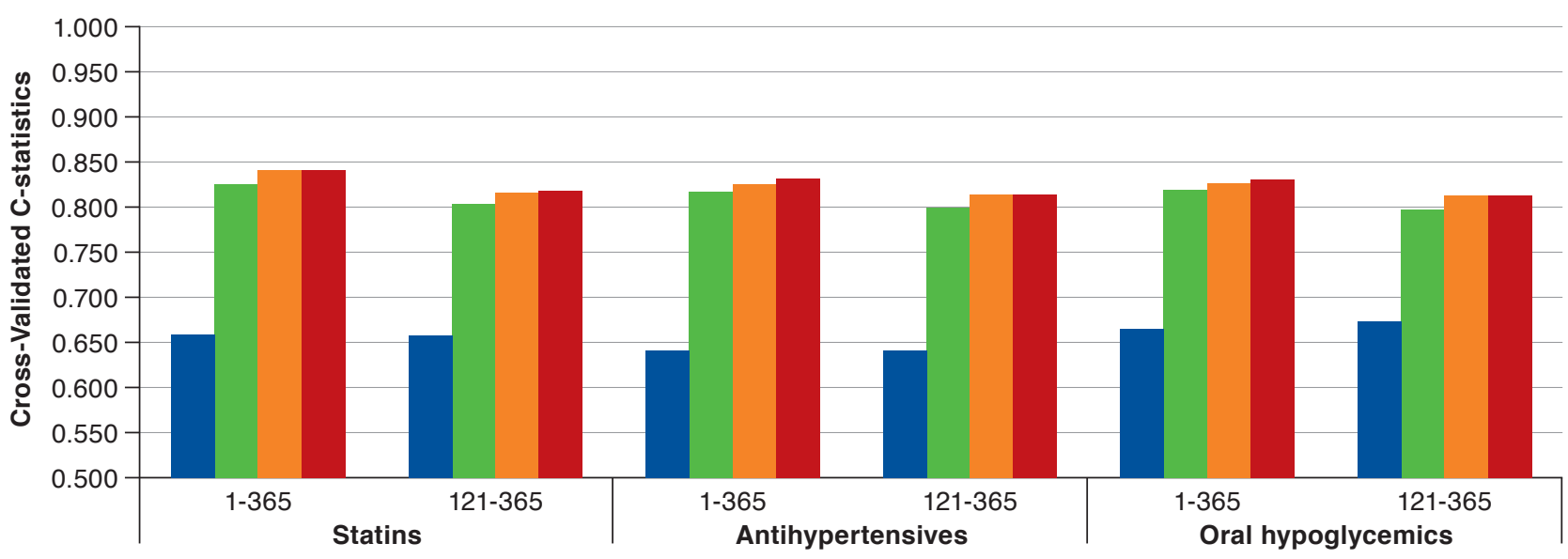

Baseline variables

Variables from days 1-120 after initiation

Baseline variables + variables from days 1-120 after initiation

All predictors 